\title{
Triamcinolone with Vitamin D Synergistic Efficacy in Psoriasis
}

\section{Background:}

- Psoriasis is one of the most common skin conditions in the United States affecting more than 8 million people.

High amounts of vitamin D has been shown to be effective in treatment of psoriasis. It also

has a well-documented safety profile at the doses and duration that will be used during this study.

- Triamcinolone and other topical corticosteroids are considered a first line treatment for mild to moderate psoriasis with a welldocumented safety profile.

Individually both of these medications have shown effectiveness in the

management and treatment of mild to moderate psoriasis.

- This study is designed to test whether a combination therapy of topical $0.1 \%$ triamcinolone with 40,000 IU vitamin D3 daily is effective in treating mild to moderate psoriasis.

\section{Methods:}

- To test this, we planned to enroll up to 24 subjects whom are age 18 and older with mild to moderate psoriasis $(2 \%+$ PASI).

- Currently, we have 7 subjects enrolled These subjects will be treated with topical $0.1 \%$ triamcinolone and oral 40,000 IU vitamin D3.

- The patients were monitored monthly until completion of trial at 6 months.

- During the 6-month period, patients had monthly blood draws (CBC, CMP, iPTH, 25hydroxyvitamin $\mathrm{D}, 1,25$ dihydroxyvitamin D), PASI scores (self-calculated), sPGA (self-calculated), Quality of Life assessment (DLQI), PHQ9 , vital signs, height and weight

\section{Results:}

- Preliminary analysis of 5 subjects showed a consistent decrease of PASI score from initial screening appointment (average score of 6.42) to final appointment on week 24 (average score of 4.72).

There was a modest decrease in SPGA for all subjects with an average starting sPGA of 2.6 and an average final sPGA of 2

- There was a modest improvement in PHQ9 for all patients with a starting average score of 6 and a final average score of 3 . Further, there was a mild improvement in DLQI score with a starting average score of 3 and a final average score of 2.25 .

- From a lab value perspective, there were no patients with recorded hypercalcemia or other recorded electrolyte or metabolic derangements throughout the duration of the study. (average Calcium: 9.5).

There was a moderate average increase in 25hydroxyvitamin $D$ levels (starting average value: 24 , final average value: 68 ). Finally, there was a modest decrease in average $\mathrm{IPTH}$ with an average starting value of 50.6 and an average final value of 23

\begin{tabular}{|c|c|c|c|c|}
\hline Patient & $\begin{array}{l}\text { PASI } \\
\text { Start }\end{array}$ & $\begin{array}{l}\text { PASI } \\
\text { Finish }\end{array}$ & $\begin{array}{l}\text { SPGA } \\
\text { Start }\end{array}$ & $\begin{array}{l}\text { SPGA } \\
\text { Finish }\end{array}$ \\
\hline 1 & 12.8 & 5.8 & 3 & 3 \\
\hline 2 & 4 & 1.6 & 2 & 1 \\
\hline 3 & 7.5 & 4.6 & 3 & 2 \\
\hline 4 & 3.6 & 4.4 & 3 & 2 \\
\hline 5 & 4.2 & 7.2 & 2 & 2 \\
\hline Average & 6.42 & 4.72 & 2.6 & 2 \\
\hline
\end{tabular}

Conclusions:

- Vitamin D combined with triamcinolone showed a decrease in PASI and SGA in several patients with psoriasis.

- While preliminary results are encouraging, more subjects are needed to make definitive conclusions on the efficaciousness of vitamin D combined with triamcinolone in patients with mild to moderate psoriasis.
Acknowledgements:

- To the BSOM Symposium for giving me the opportunity to share my research.

- To the PTU department for their continued support and assistance in recruiting and managing subjects.

Disclosures:

There are no disclosures

for this project or presentation. 